\title{
Earthquake probability based on multidisciplinary observations with correlations
}

\author{
Masajiro Imoto \\ National Institute for Earth Science and Disaster Prevention, \\ 3-1 Ten-nodai, Tsukuba-shi, Ibaraki 305-0006, Japan \\ (Received October 4, 2005; Revised February 14, 2006; Accepted July 21, 2006; Online published December 25, 2006)
}

\begin{abstract}
A number of researchers have formulated earthquake probabilities based on precursory anomalies of multidisciplinary observations in which the underlying assumption is that the occurrence of one precursory anomaly is independent from those of other kinds of anomalies. Observations were classified into two groups, those events followed by an earthquake and those that were not, and the ratio of observed precursors in both groups was taken into consideration. In the present report, recent advances in statistical seismology are considered within the framework of these earthquake probabilities, and the formulations are extended to cases in which precursory anomalies are observed as continuous measurements. The effects originating from mutual correlations between two precursory anomalies are also considered. It is assumed that observed values of each discipline follow a normal distribution, either as a group of observations followed by an earthquake (conditional density distribution) or as a group of observations not followed by an earthquake (background density distribution). Special attention is given to the case in which two kinds of observations are correlated in the conditional density distribution but not in the background density distribution. The results obtained are compared with cases in which the observations are independent of each other in both distributions. The geometrical mean of the probability gain is greater in the correlated case than in the independent case and becomes infinitely large when the absolute value of the correlation coefficient approaches one. This finding enables a wider application of earthquake probability than has been previously possible based on multidisciplinary observations.
\end{abstract}

Key words: Earthquake probability, multidisciplinary observation, correlation, information gain, KullbackLeibler information quantity.

\section{Introduction}

Utsu (1977, 1982), Aki (1981), and a number of others (Hamada, 1983; Grandori et al., 1988) have formulated an earthquake probability for the simultaneous detection of multiple precursory phenomena with empirical relations of each phenomenon. The underlying assumption in the formulations is that different precursory phenomena occur independently of each other. In such a case, the probability expected from detecting multiple precursory phenomena is given by a product of probability gains for the respective observations and the probability estimated from secular seismicity. Therefore, the probability could become large when multiple precursory phenomena are detected simultaneously. This formula has been practically applied for estimating the probabilities of characteristic earthquakes in California (Jones, 1985) and Japan (Imoto, 2004), where a renewal process model and a model of foreshocks represent two different precursory phenomena independent of each other. With the exception of these two cases, no other examples have been reported since it is widely believed that no reliable precursors have been observed except for only a few phenomena such as foreshocks (Wyss and Booth,

Copyright (c) The Society of Geomagnetism and Earth, Planetary and Space Sciences (SGEPSS); The Seismological Society of Japan; The Volcanological Society of Japan; The Geodetic Society of Japan; The Japanese Society for Planetary Sciences; TERRAPUB.
1997; Geller, 1997). This is partly due to difficulties in the systematic investigation necessary for the statistical estimation of each precursory phenomenon in order that this formula be applied, although there have been a large number of reports of multiple precursory phenomena for individual earthquakes (e.g. Tsunogai and Wakita, 1996; Yamada and Oike, 1996, Hayakawa et al., 1996; Katao et al., 1997). However, if we define precursors in a general sense as those providing information on earthquake occurrences, we can discover additional precursory parameters that could be useful for estimating earthquake probabilities. The $a$ - and $b$-values in the Gutenberg-Richter relation, for example, would be considered one such generalized precursory parameter (Wiemer and Wyss, 1997; Zuñiga and Wyss, 2001). Another example can be taken from the case of changes in mean event size (Imoto, 2003). These examples suggest that reliable precursor parameters in the general sense can be listed more often than we had originally thought.

Other problems in applying this formula may arise from the assumption that precursory phenomena appear to be independent of each other. First, if multiple precursory phenomena were to occur independently of each other, the ratio of the number of the earthquakes preceded by all types of precursory phenomena to the total number of target earthquakes-which is called reliability (Reasenberg and 
Matthews, 1988)—will become infinitesimally smaller as more precursory phenomena are combined. This would mean that earthquakes of high probability would be forecasted only scarcely. Next, if we consider that various precursory phenomena occur during a preparation stage of an earthquake, their occurrences may not appear to be independent of each other, even though the observed phenomena are different. It may be possible that we are observing different phenomena occurring due to a single process or that we are viewing phenomena in a causality relationship as an independent observation. In addition to the above points, the formula should be modified based on continuous functions handling generalized precursors, where phenomena are treated as two categorical values - that is, as the appearance or non-appearance of a precursor. The appearance of the precursor is usually judged from observed physical quantities, and the probability is calculated for each instance. We are, in fact, cutting off part of the information by categorizing observed multiple states or continuously varying quantities into two categories. If we were to provide a probability, we do not necessarily limit ourselves to the two categories.

From these viewpoints, there may be room for a fundamental reconsideration of the formulations of probability based on multidisciplinary observations. By handling the phenomena with a quantitative observed value, we may be able to avoid cutting off information. Also, recent studies of the point process analysis in statistical seismology are quite suggestive that an earthquake probability model should be formulated through an empirical approach. In the present report, the two categorical phenomena handled by the early studies are first extended to phenomena with multiple categories. The problems are subsequently clarified by expressing the probability gain with the quantity of information. Based on these steps, the case of two observed phenomena that are assumed to be correlated only when an earthquake occurs is observed, and the effect of dependence (having correlation) over the probability gain is evaluated analytically.

\section{Information Gain for Discrete Observations}

In general, earthquake probability is discussed here in a particular time and space. However, for the sake of simplicity, the discussion is limited to only the time axis. Let there be $N_{0}$ small time intervals of the length $\Delta t$ in the period for this study. Earthquakes occurred in $m_{0}$ intervals but did not occur in $n_{0}$ intervals. Assume NA levels of earthquake probability for each interval, which corresponds to NA states of observed values $A_{i}(i=1,2, \ldots, N A)$, defined by observation prior to the start of the interval. The earthquake probability will be discussed using the NA different groups of intervals. The numbers of intervals in the $i$-th level of probability are designated as $m_{i}$ (including an earthquake) and $n_{i}$ (not including an earthquake). Let there be $\sum m_{i}=m_{0}, \sum n_{i}=n_{0}$, and $m_{0}+n_{0}=N_{0}$. The probability that $A_{i}$ is observed provided the prior condition that an earthquake occurs in the following interval, $P\left(A_{i} \mid E\right)$, is given by $m_{i} / m_{0}$, and the probability that $A_{i}$ is observed provided that an earthquake does not occur in $P\left(A_{i} \mid \bar{E}\right)$ is expressed as $n_{i} / n_{0}$. Therefore, the probability that an earthquake occurs when $A_{i}$ is observed in $P\left(E \mid A_{i}\right)$ is

$$
\begin{aligned}
P\left(E \mid A_{i}\right) & =\frac{P\left(A_{i} \mid E\right) P(E)}{P\left(A_{i} \mid E\right) P(E)+P\left(A_{i} \mid \bar{E}\right) P(\bar{E})} \\
& =\frac{m_{i}}{m_{i}+n_{i}}
\end{aligned}
$$

by Bayes theorem.

Conversely, the probability that an earthquake does not occur is

$$
P\left(\bar{E} \mid A_{i}\right)=\frac{n_{i}}{m_{i}+n_{i}} .
$$

In such a case, the likelihood is given by the following formula

$$
L=\prod_{i=1}^{N A} P\left(E \mid A_{i}\right)^{m_{i}} \cdot \prod_{i=1}^{N A} P\left(\bar{E} \mid A_{i}\right)^{n_{i}} .
$$

Substituting Eqs. (1) and (2) into Eq. (3) leads to

$$
L=\prod_{i=1}^{N A}\left(\frac{m_{i}}{m_{i}+n_{i}}\right)^{m_{i}} \cdot \prod_{i=1}^{N A}\left(\frac{n_{i}}{m_{i}+n_{i}}\right)^{n_{i}} .
$$

Taking the logarithm of Eq. (4) gives

$$
\begin{aligned}
\ln L & =\sum_{i=1}^{N A} m_{i} \ln \frac{m_{i}}{n_{i}+m_{i}}+\sum_{i=1}^{N A} n_{i} \ln \frac{n_{i}}{n_{i}+m_{i}} \\
& \approx \sum_{i=1}^{N A} m_{i} \ln \frac{m_{i}}{n_{i}}-\sum_{i=1}^{N A} n_{i}\left(\frac{m_{i}}{n_{i}}\right) \\
& =\sum_{i=1}^{N A} m_{i} \ln \frac{m_{i}}{n_{i}}-\sum_{i=1}^{N A} m_{i} \\
& =-m_{0}+\sum_{i=1}^{N A} m_{i} \ln \frac{m_{i}}{n_{i}},
\end{aligned}
$$

where we assume $n_{i} \gg m_{i}$. Hereafter, ln refers to the natural logarithm.

If we consider earthquake occurrence to be a stationary Poisson process, the logarithm of its likelihood, $L_{0}$, is

$$
\ln L_{0}=-m_{0}+m_{0} \ln \frac{m_{0}}{N_{0}} .
$$

Therefore, the difference in log-likelihood between both cases, i.e., the information gain (denoted by $I G$ ) (VereJones, 1998; Daley and Vere-Jones, 2003), is

$$
I G=\ln L-\ln L_{0}=\sum_{i=1}^{N A} m_{i} \cdot \ln \frac{\frac{m_{i}}{n_{i}}}{\frac{m_{0}}{N_{0}}} .
$$

The average value of $I G$ for an event (denoted by $I G p e$ ) (Imoto, 2004), is

$$
I G p e=\frac{1}{m_{0}}\left(\ln L-\ln L_{0}\right)=\sum_{i=1}^{N A} \frac{m_{i}}{m_{0}} \ln \frac{\frac{m_{i}}{m_{0}}}{\frac{n_{i}}{N_{0}}} .
$$

Replacing $m_{i} / m_{0}$ and $n_{i} / N_{0}$ with $p a_{i}$ and $q a_{i}$, respectively, Eq. (8) becomes

$$
I G p e=\sum_{i=1}^{N A} p a_{i} \ln \frac{p a_{i}}{q a_{i}} .
$$


Where $p a_{i}$ and $q a_{i}$ are expressing probabilities of observing $A_{i}$ in intervals prior to an interval including an earthquake and not including one, respectively. Equation (9) represents that IGpe is equivalent to the Kullback-Leibler quantity of information (Sakamoto et al., 1983) expressing the distance between the two probability distributions.

There is another observation $B$ that should be considered further. Let us assume there are $N B$ state values $B_{j}(j=$ $1,2, \ldots, N B)$ in observation item $B$. In a similar way to observation $A, p b_{j}$ and $q b_{j}$ are expressing probabilities of observing $B_{j}$ prior to an interval with an earthquake and without one, respectively. Let us connote the conditioned probabilities that $A_{i}$ and $B_{j}$ are observed simultaneously as $p_{i j}$ and $q_{i j}$. The IGpe value corresponding with those probability distributions, $I G p e(A, B)$, is expressed with the following equation.

$$
I G p e(A, B)=\sum_{i=1}^{N A} \sum_{j=1}^{N B} p_{i j} \ln \frac{p_{i j}}{q_{i j}} .
$$

If observations $A$ and $B$ are conditionally independent,

$$
\begin{aligned}
& p_{i j}=p a_{i} \times p b_{j} \\
& q_{i j}=q a_{i} \times q b_{j}
\end{aligned}
$$

stands, and the IGpe value becomes

$$
\begin{aligned}
I G p e(A, B) & =\sum_{i=1}^{N A} \sum_{j=1}^{N B} p a_{i} p b_{j} \ln \frac{p a_{i} p b_{j}}{q a_{i} q b_{j}} \\
& =\sum_{i=1}^{N A} p a_{i} \ln \frac{p a_{i}}{q a_{i}}+\sum_{j=1}^{N B} p b_{j} \ln \frac{p b_{j}}{q b_{j}} \\
& =I G p e(A)+I G p e(B) .
\end{aligned}
$$

Since the value is equivalent to the logarithm of geometric mean of probability gain (Imoto, 2001), Eq. (12) is an extension of the formula given by Aki (1981), in that probability gains based on two independent observations are given by a product of each probability gain.

\section{Information Gain for Continuous Distribution}

It is assumed here that the observed value $x$ of observation $A$ is a continuous variable. It is also assumed that the density distribution of $x, f_{1}(x)$ can be obtained from the value observed in a moment immediately before a target earthquake (the conditional density distribution) and that the density distribution expressed by $g_{1}(x)$ can be obtained by observing a section not followed by any target earthquake (the background density distribution). Also, for observation $B$, the assumption is made that distributions $f_{2}(y)$ and $g_{2}(y)$ can be obtained for observed value $y$. Furthermore, distributions $f(x, y)$ and $g(x, y)$ are assumed to be obtained from simultaneous observation of $A$ and $B$ for the conditional density and background density distributions, respectively. In this case, these can be expressed as follows in accordance with Eqs. (9) and (10) obtained in the previous section.

$$
I G p e(A)=\int f_{1}(x) \ln \frac{f_{1}(x)}{g_{1}(x)} d x
$$

$$
\begin{aligned}
& I G p e(B)=\int f_{2}(y) \ln \frac{f_{2}(y)}{g_{2}(y)} d y \\
& I G p e(A, B)=\iint f(x, y) \ln \frac{f(x, y)}{g(x, y)} d x d y .
\end{aligned}
$$

If it is assumed here that $x$ and $y$ are independent of each other in the conditional density distribution and background density distribution, the following equations hold:

$$
\begin{aligned}
& f(x, y)=f_{1}(x) f_{2}(y) \\
& g(x, y)=g_{1}(x) g_{2}(y) .
\end{aligned}
$$

In this case, Eq. (14) can be rewritten as follows:

$$
\begin{aligned}
I G p e & (A, B) \\
& =\iint f_{1}(x) f_{2}(y)\left\{\ln \frac{f_{1}(x)}{g_{1}(x)}+\ln \frac{f_{2}(y)}{g_{2}(y)}\right\} d x d y \\
& =\int f_{1}(x) \ln \frac{f_{1}(x)}{g_{1}(x)} d x+\int f_{2}(y) \ln \frac{f_{2}(y)}{g_{2}(y)} d y \\
& =I G p e(A)+I G p e(B) .
\end{aligned}
$$

This equation corresponds to Eq. (12) in the previous section. Equations (13), (14), and (17) are interpreted as the Kullback-Leibler quantity of information (Sakamoto et al., 1983), which represents the distance between the conditional density distribution and background density distribution (Imoto, 2004).

\section{Two-Dimensional Normal Distribution}

In this section, we examine the case in which both the conditional density and background density distributions can be expressed as a normal distribution in two observation items, $A$ and $B$. We compare the Kullback-Leibler quantity of information between the case in which their observed values $x$ and $y$ are independent of each other and that in which they are correlated.

\subsection{Independent case}

For observation item $A$, conditional density distribution $f_{1}(x)$ and background density distribution $g_{1}(x)$ can be expressed by the following equations:

$$
\begin{aligned}
& f_{1}(x)=\frac{1}{\sqrt{2 \pi} \sigma_{x}} e^{-\frac{\left(x-x_{0}\right)^{2}}{2 \sigma_{x}^{2}}} \sim N\left(x_{0}, \sigma_{x}^{2}\right) \\
& g_{1}(x)=\frac{1}{\sqrt{2 \pi}} e^{-\frac{x^{2}}{2}} \sim N(0,1) .
\end{aligned}
$$

Here, by selecting an appropriate unit and origin of variable $x$, Eq. (19) can be satisfied in general. In this case, the IGpe value for observation $A, I G p e(A)$, can be represented as follows (Sakamoto et al., 1983):

$$
\begin{aligned}
\operatorname{IGpe}(A)= & \int_{-\infty}^{\infty} \frac{1}{\sqrt{2 \pi} \sigma_{x}} e^{-\left(x-x_{0}\right)^{2} / 2 \sigma_{x}^{2}} \\
& \times\left[\ln \left\{\frac{1}{\sqrt{2 \pi} \sigma_{x}} e^{-\left(x-x_{0}\right)^{2} / 2 \sigma_{x}^{2}}\right\}\right. \\
& \left.-\ln \left\{\frac{1}{\sqrt{2 \pi}} e^{-\frac{x^{2}}{2}}\right\}\right] d x \\
= & \frac{1}{2} \ln \frac{1}{\sigma_{x}^{2}}+\frac{\sigma_{x}^{2}+x_{0}^{2}-1}{2} .
\end{aligned}
$$


Also, for observation $B$, it is assumed that two distributions, $f_{2}(y)$ and $g_{2}(y)$, are represented by the following normal distributions:

$$
\begin{aligned}
& f_{2}(y) \sim N\left(y_{0}, \sigma_{y}^{2}\right) \\
& g_{2}(y) \sim N(0,1) .
\end{aligned}
$$

The following equation can be obtained in the same manner as for observation $A$ :

$$
\operatorname{IGpe}(B)=\frac{1}{2} \ln \frac{1}{\sigma_{y}^{2}}+\frac{\sigma_{y}^{2}+y_{0}^{2}-1}{2} .
$$

If it is assumed that $x$ and $y$ are independent in both the conditional density and background density distributions, the following equation can be obtained based on Eq. (17):

$$
I G p e(A, B)=\frac{1}{2} \ln \frac{1}{\sigma_{x}^{2} \sigma_{y}^{2}}+\frac{\sigma_{x}^{2}+\sigma_{y}^{2}}{2}+\frac{x_{0}^{2}+y_{0}^{2}}{2}-1 .
$$

Figure 1 illustrates the case of $x_{0}=2, y_{0}=1, \sigma_{x}=$ $\sigma_{y}=1$. Figure 1(a) presents the contour diagrams of the probability density of $f(x, y)$ and $g(x, y)$, while Figs. 1(b) and $1(\mathrm{c})$ depict their marginal density distributions, $f_{1}(x)$, $g_{1}(x)$, and $f_{2}(y), g_{2}(y)$. From Eq. (24), it can be seen that the IGpe value is derived from the positional difference between $f(x, y)$ and $g(x, y)$. Figures 1(d) and 1(e) display the marginal density distributions for the $x^{\prime}-y^{\prime}$ coordinate system, where the $x^{\prime}$ coordinate passes through the origin of the $x-y$ coordinate system. When viewed on the $x^{\prime}$ coordinate system, the distance between $f_{1}^{\prime}\left(x^{\prime}\right)$ and $g_{1}^{\prime}\left(x^{\prime}\right)$ has the relation, $x_{0}^{\prime 2}=x^{2}+y^{2}$. The contribution from this distance is the IGpe value itself. When viewed on the $y^{\prime}$ coordinate system, $f_{2}^{\prime}\left(y^{\prime}\right)$ and $g_{2}^{\prime}\left(y^{\prime}\right)$ virtually overlap each other, so the contribution of this component to the IGpe value is 0 .

\subsection{Correlated conditional distribution}

It is assumed here that the correlation between $x$ and $y$ with a correlation coefficient of $\rho$ can be observed only in the conditional density distribution $f(x, y)$. If it is assumed that $f_{1}(x)$ and $f_{2}(y)$ are obtained through observation as the marginal density distribution of $x$ and $y$, the covariance matrix, $\operatorname{Cov}(x, y)$ can be expressed as follows:

$$
\operatorname{Cov}(x, y)=\left(\begin{array}{cc}
\sigma_{x}^{2} & \rho \sigma_{x} \sigma_{y} \\
\rho \sigma_{x} \sigma_{y} & \sigma_{y}^{2}
\end{array}\right) .
$$

The coordinate axes are then shifted in parallel to the point $\left(x_{0}, y_{0}\right)$ and are rotated by angle $\varphi$. Here, it is assumed that $\varphi$ satisfies the following equation:

$$
\tan 2 \varphi=\frac{2 \rho \sigma_{x} \sigma_{y}}{\sigma_{x}^{2}-\sigma_{y}^{2}}
$$

If the coordinate after the rotation is denoted as $x^{\prime}, y^{\prime}$, the following equation will be obtained:

$$
\operatorname{Cov}\left(x^{\prime}, y^{\prime}\right)=\left(\begin{array}{cc}
\lambda_{1}^{2} & 0 \\
0 & \lambda_{2}^{2}
\end{array}\right) \text {. }
$$

Here,

$$
\begin{aligned}
& \lambda_{1}^{2}=\sigma_{x}^{2} \cos ^{2} \varphi+\sigma_{y}^{2} \sin ^{2} \varphi+2 \rho \sigma_{x} \sigma_{y} \sin \varphi \cos \varphi \\
& \lambda_{2}^{2}=\sigma_{x}^{2} \sin ^{2} \varphi+\sigma_{y}^{2} \cos ^{2} \varphi-2 \rho \sigma_{x} \sigma_{y} \sin \varphi \cos \varphi .
\end{aligned}
$$
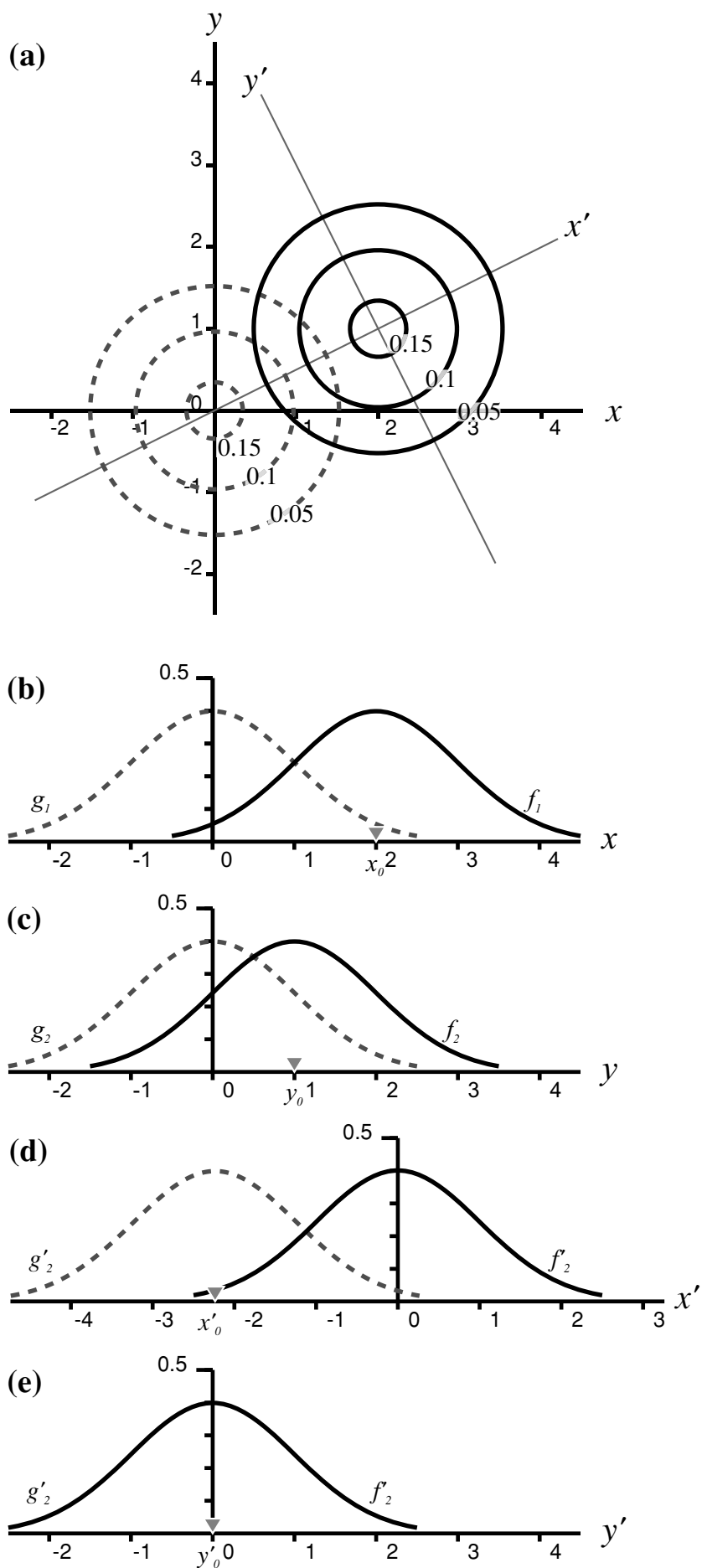

Fig. 1. Schematic of the conditional probability density and background probability density distributions where variables $x$ and $y$ are independent of each other in both distributions. (a) Contours of iso-probability densities for the conditional density distribution (solid lines) and background distribution (dashed lines). (b) Marginal distributions of variable $x$ for the conditional density distribution (solid lines) and background distribution (dashed lines). (c) Same as in Fig. 1(b) but for variable $y$. (d) Same as in Fig. 1(b) but for variable $x^{\prime}$. (e) Same as Fig. 1(b) but for variable $y^{\prime}$. See the text for the parameters of the distributions.

In this case, the following equations will be satisfied:

$$
\begin{aligned}
& \lambda_{1}^{2}+\lambda_{2}^{2}=\sigma_{x}^{2}+\sigma_{y}^{2} \\
& \lambda_{1}^{2} \cdot \lambda_{2}^{2}=\sigma_{x}^{2} \cdot \sigma_{y}^{2}\left(1-\rho^{2}\right) .
\end{aligned}
$$


After the shift and rotation of the coordinate axes, $f\left(x^{\prime}, y^{\prime}\right)$ and $g\left(x^{\prime}, y^{\prime}\right)$ can be expressed by the following equation:

$$
f\left(x^{\prime}, y^{\prime}\right)=\frac{1}{2 \pi \lambda 1 \lambda 2} e^{-\frac{x^{\prime 2}}{2 \lambda_{1}^{2}}-\frac{y^{\prime 2}}{2 \lambda_{2}^{2}}}
$$

and

$$
g\left(x^{\prime}, y^{\prime}\right)=\frac{1}{2 \pi} e^{-\frac{\left(x^{\prime}-x_{0}^{\prime}\right)^{2}}{2}-\frac{\left(y^{\prime}-y_{0}^{\prime}\right)^{2}}{2}} .
$$

Here,

$$
x_{0}^{\prime 2}+y_{0}^{\prime 2}=x_{0}^{2}+y_{0}^{2} .
$$

Therefore, for the case in which the variables $x$ and $y$ in the conditional density distribution are correlated for each other with a correlation coefficient of $\rho$, the IGpe value, $I G p e(A, B \mid \rho)$, can be obtained from the following equation by substituting $\lambda_{1}$ and $\lambda_{2}$ for $\sigma_{1}$ and $\sigma_{2}$ into Eq. (24) and using Eqs. (29), (30) and (33):

$$
\begin{aligned}
\operatorname{IGpe}(A, B \mid \rho)= & \frac{1}{2} \ln \frac{1}{\lambda_{1}^{2} \lambda_{2}^{2}}+\frac{\lambda_{1}^{2}+\lambda_{2}^{2}}{2}+\frac{x_{0}^{\prime 2}+y_{0}^{\prime 2}}{2}-1 \\
= & \frac{1}{2} \ln \frac{1}{\sigma_{x}^{2} \sigma_{y}^{2}\left(1-\rho^{2}\right)}+\frac{\sigma_{x}^{2}+\sigma_{y}^{2}}{2} \\
& +\frac{x_{0}^{2}+y_{0}^{2}}{2}-1 .
\end{aligned}
$$

The difference in IGpe, $\Delta I G p e$, can then be expressed as follows between the case where there is a correlation between $x$ and $y$ and the case where there is no correlation between them:

$$
\begin{gathered}
\operatorname{IGpe}(A, B \mid \rho)-I G p e(A, B \mid \rho=0) \\
=\frac{1}{2} \ln \frac{1}{1-\rho^{2}} \geq 0 .
\end{gathered}
$$

Therefore, if two phenomena are occurring independently of each other in the background density distribution, but are correlated with each other in the conditional density distribution, the resultant mean probability gain always exceeds that of the independent case, as given by the product of the probability gains obtained from the respective phenomena.

We can understand the formulation visually in a simple example. Figure 2 illustrates the case of $x_{0}=2, y_{0}=1$, $\sigma_{x}=\sigma_{y}=1, \rho=0.8$. These values are the same as those shown in Fig. 1 except for $\rho=0.8$, so the contribution of $\rho=0.8$ to the IGpe value should be clear. Figure 2(a) plots the contours of probability densities of $f(x, y)$ and $g(x, y)$, and their marginal density distributions of $x$ and $y$ are essentially equal to those shown in Figs. 1(b) and 1(c). Therefore, it can be understood that only the positional difference between $f(x, y)$ and $g(x, y)$ contributes to the value in this state. Marginal density distributions of $x^{\prime}$ and $y^{\prime}$ obtained by the above coordinate transformation are presented in Figs. 2(b) and 2(c). The positional differences between $f_{1}^{\prime}\left(x^{\prime}\right)$ and $g_{1}^{\prime}\left(x^{\prime}\right)$ and between $f_{2}^{\prime}\left(y^{\prime}\right)$ and $g_{2}^{\prime}\left(y^{\prime}\right)$ contribute to the IGpe value equivalently to those in Figs. 1(b) and 1(c) since $x_{0}^{\prime 2}+y_{0}^{\prime 2}=x_{0}^{2}+y_{0}^{2}$. In addition, the difference in shape between $f_{1}^{\prime}\left(x^{\prime}\right)$ and $g_{1}^{\prime}\left(x^{\prime}\right)$ and between $f_{2}^{\prime}\left(y^{\prime}\right)$ and $g_{2}^{\prime}\left(y^{\prime}\right)$ contributes to the $I G p e$ value. The IGpe value accounting for the correlation coefficient becomes larger than the sum of the IGpe values calculated (a)
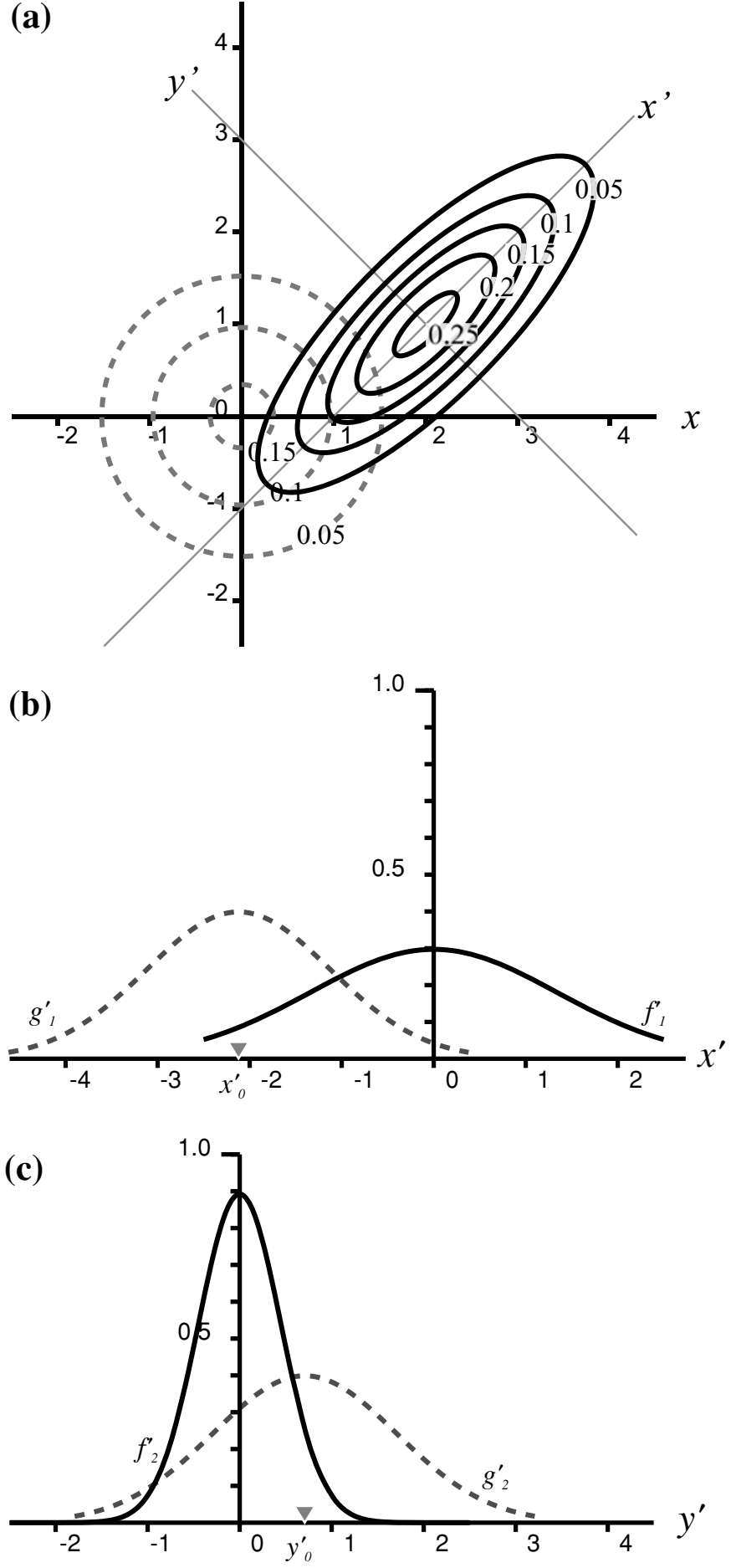

Fig. 2. Schematic of the conditional probability density and background probability density distributions where variables $x$ and $y$ are independent in the background density distribution but are positively correlated with each other in the conditional distributions. (a) Contours of iso-probability densities for the conditional density distribution (solid lines) and background distribution (dashed lines). (b) Marginal distributions of variable $x^{\prime}$ for the conditional density distribution (solid lines) and background distribution (dashed lines). (c) Same as Fig. 2(b) but for variable $y^{\prime}$. See the text for the parameters of the distributions.

separately as the marginal density distributions of $x$ and $y$. Figure 3 is the same as Fig. 2 except for the negative correlation $\rho=-0.8$. Here, the marginal distributions of $x^{\prime}$ 


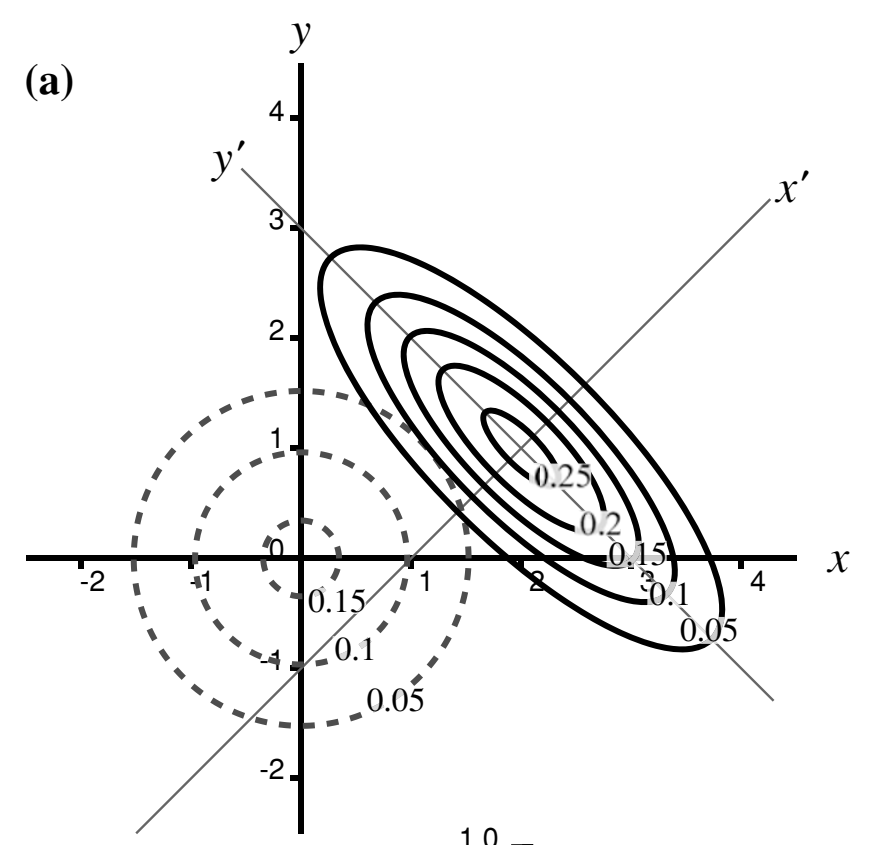

(b)

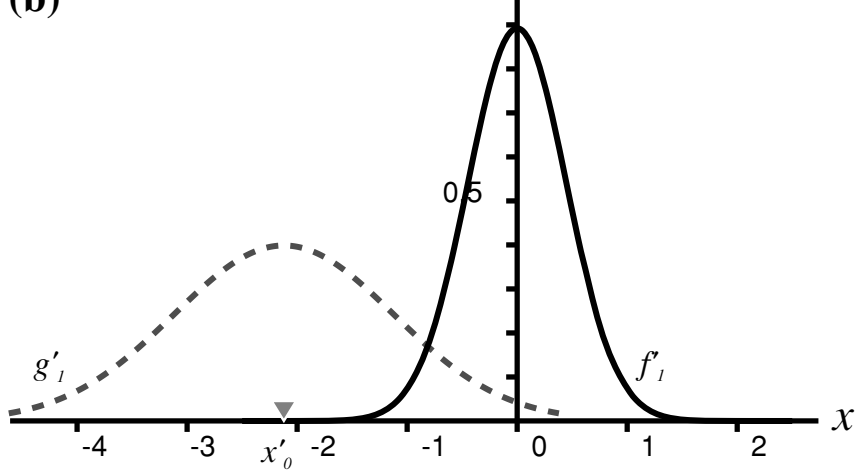

(c)

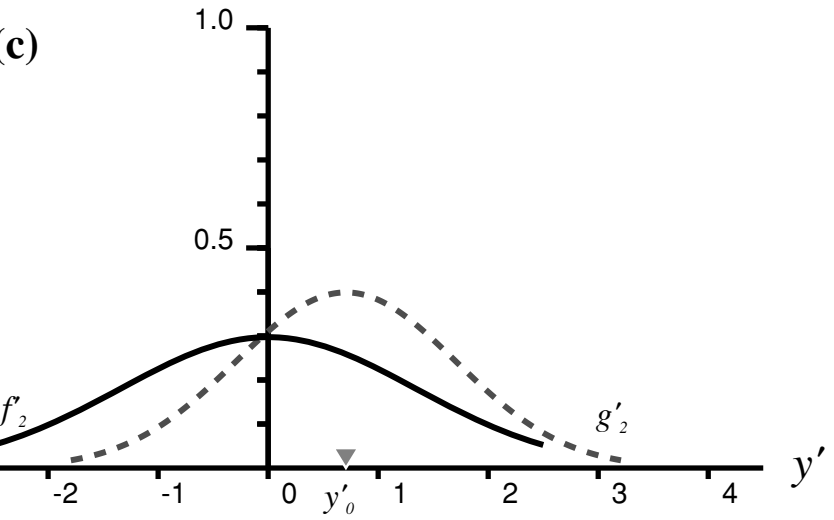

Fig. 3. Same as in Fig. 2 but for the negative correlation in the conditional distributions.

and $y^{\prime}$ are illustrated in Figs. 3(b) and 3(c). It can be easily seen that the differences in the shape of the function and in its location between the conditional and background distributions contribute to the IGpe value as they did for the positive correlation. In more general cases, where $\sigma_{x}$ and $\sigma_{y}$ are not equal to 1 , it could be understood through a similar consideration that the difference in the sense of the correlation coefficient is unrelated to the IGpe value.

Figure 4 depicts the value of Eq. (35) using the coefficient of correlation as a variable within the range of $0<|\rho|<$

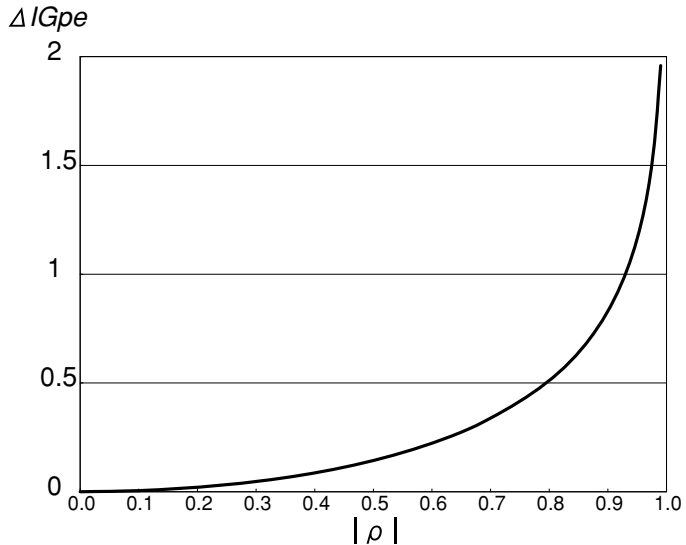

Fig. 4. Variation of $\Delta I G p e$ with the absolute value of $\rho$, the correlation coefficient in the conditional distribution. $\Delta I G p e$ is calculated as the difference in IGpe between the cases with and without correlation in the conditional density distribution.

0.99. As the absolute value of the correlation coefficient approaches 1 , the effect of the correlation in the conditional distribution contributes greatly to the increase in the $I G p e$ value. In other words, a larger probability gain can be obtained as the correlation becomes stronger. This can be schematically understood as follows. If two phenomena $x$ and $y$ are strongly correlated in the conditional distribution, a point representing $x$ and $y$ in two-dimensional space is observed in a certain restricted ellipse area with a high probability. However, for the background distribution, the point of $x$ and $y$ can be observed with only an extremely low probability. Thus, when we observe a set of $(x, y)$ in (out of) that small area, we can expect a high (low) probability of an earthquake. A large $I G p e$ value implies that this expectation is much more effective than when $x$ and $y$ are independent of each other in both the conditional and background distributions.

\section{Discussion and Summary}

In our definition, the $a$ - and $b$-values in the GutenbergRichter relation can become precursors in the broad sense. By using these parameters, a hazard varying with time is defined as

$$
\lambda\left(t \mid M \geq M_{o}\right)=10^{a-b M_{o}},
$$

where only earthquakes with magnitudes of $M_{o}$ and larger as targets are considered for estimating probability. The parameter values are implicitly functions of time since sample earthquakes for evaluation would change from time to time. Consequently, for an estimation of earthquake probability to be used in the future, it should be evaluated with those occurring prior to the time of assessment. By evaluating the average values of the $a$ - and $b$-values as $a_{0}$ and $b_{0}$, Eq. (36) is represented by

$$
\lambda\left(t \mid M \geq M_{o}\right)=10^{a_{0}-b_{0} M_{o}} \cdot 10^{\left(a-a_{0}\right)} \cdot 10^{-\left(b-b_{0}\right) M_{o}} .
$$

The above hazard can be considered to be the value obtained by a multiproduct of three terms; that is

$\lambda\left(t \mid M \geq M_{o}\right)=\lambda_{0}\left(a_{0}, b_{0}\right) \cdot \mu_{1}\left(a-a_{0}\right) \cdot \mu_{2}\left(b-b_{0}\right)$. 
The first term on the right side expresses a hazard invariant with time. The second and third terms are like probability gains evaluated for each phenomenon in Aki's formula. A hazard function based on the Gutenberg-Richter relation can thus be considered to be an example of Aki's formula, and widely accepted studies (Wiemer and Wyss, 1997; Zuñiga and Wyss, 2001; Schorlemmer et al., 2003) in this direction have implicitly assumed that the two parameters of $a$ - and $b$-values can be considered to be independent of each other. Based on the viewpoints of the present study, careful studies have to be made in each case in order to draw a conclusion on this point; these are beyond the scope of the present study and should be performed separately in the future. Even if the two parameters of the $a$ and $b$-values were independent of each other in the background distribution, it may be hard to apply the present formula directly to them since distributions of these values are not likely to be expressed in a normal function. It is believed that seismological, geological, and other conditions may affect the $a$ - or $b$-values (Mogi, 1962; Scholz, 1968; Wyss, 1973; Mori and Abercrombie, 1997; Wyss and Matsumura, 2002; Schorlemmer et al., 2005). Therefore, if we consider a background distribution of them both in time and space, the shape of the distribution may seriously depend on these conditions. Even slight modifications of a study volume may require the change of a function fitted to the background distribution into a function of another type. However, if we do not consider a value of direct observation but instead a derivative of the value, the background distribution of the parameter becomes more likely to be a normal function after an appropriate transformation of the parameter.

Imoto (2003) attempted to construct a model for estimating the probabilities of moderate earthquakes in Kanto, Japan. In his modeling, temporal changes in the mean event size of earthquakes smaller than that of a target plays an important role as a precursor in the broad sense. After parameter transformation, a beta function with two free parameters is fitted to both the background and conditional distributions of the precursor parameter (denoted by $m$ in the literature). If we perform a further transformation of the parameter in such a way that the distribution of the new parameter can be well represented by a normal distribution, we can obtain one possible precursor that could be applicable to the present formula. Actually, it could be obtained through the transformation as

$$
\int_{0}^{\mu} \mathrm{B}(s \mid \alpha, \beta) d s=\int_{-\infty}^{\eta} \frac{1}{\sqrt{2 \pi}} \mathrm{e}^{-\frac{x^{2}}{2}} d x,
$$

where $B$ denotes Beta function as

$$
\begin{aligned}
& \mathrm{B}(x \mid \alpha, \beta)= \begin{cases}\frac{1}{\mathrm{~B}(\alpha, \beta)} x^{\alpha-1}(1-x)^{\beta-1} & \text { if } 0<x<1 \\
0 & \text { otherwise }\end{cases} \\
& B(\alpha, \beta)=\int_{0}^{1} x^{\alpha-1}(1-x)^{\beta-1} d x
\end{aligned}
$$

and $\alpha(>0)$ and $\beta(>0)$ denote its two parameters. The parameters of $\mu$ and $\eta$ represent the precursor parameter before and after the transformation, respectively. Figure 5 illustrates the obtained conditional and background distri-

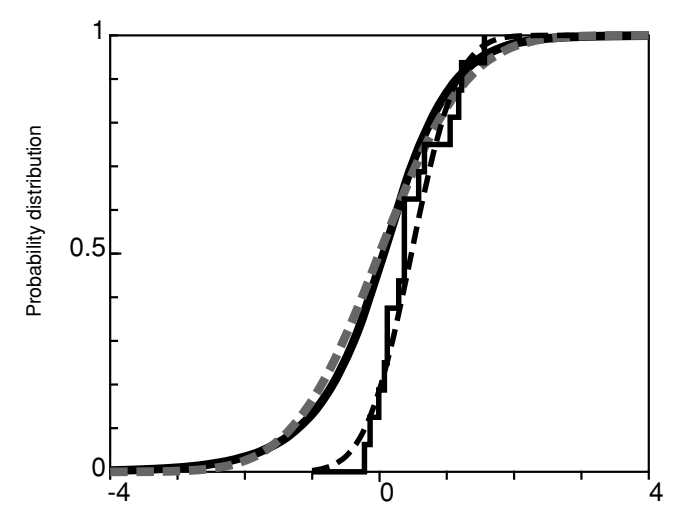

Fig. 5. The conditional and background distributions empirically obtained from an observation of temporal changes in mean event size are indicated in a step function and a solid curve. A dashed thin line and a dashed gray line indicate the normal distributions fitted to the empirical conditional and background distributions.

butions of the $\eta$ parameter and two normal distributions fitted to them. This figure indicates that the two distributions are well approximated with normal distributions. The $\eta$ parameter could therefore be listed for an application of the present formula in a future study.

When one research group proposes multidiscipline precursors, they can rather easily build these into one probability model, even if these precursors are mutually dependent on one another. However, generally speaking, one author proposes a certain precursor and a different author proposes another one for a different space and/or time. Therefore, verification of independency of multiple precursors should require simultaneous observation of the respective precursors. This process would take additional years to accumulate data to verify independency, primarily because corresponding target earthquakes do not occur so often (Evison and Rhoades, 1997; Evison and Rhoades, 1999; Imoto and Yamamoto, 2006). The present results can reduce these additional years occasionally since it may be possible to investigate independency between multiple observations in a fairly short time for the background distribution. When multiple precursors are proven to be mutually independent for the background distribution, Eq. (35) suggests that a combined model assuming a correlation coefficient of 0 would estimate IGpe of the lowest limit among all the values of possible correlations. This should still be better than an IGpe estimated from each precursory phenomenon. Thus, the formula obtained in the present study could play an important role in an early stage of constructing a combined model.

In summary, assuming normal distributions as an empirical distribution for observations of two different precursors in a broad sense, we analytically evaluate an effect caused by a correlation in the conditional distribution between two precursors. If the two parameters of the precursors are independent in the background distribution and correlated in the conditional distribution, the mean probability gain becomes larger than that in the independent (uncorrelated) case in the conditional distribution. This effect becomes remarkable with an absolute value of the correlation coefficient approaching 1 . 
Acknowledgments. The author would like to express his thanks to D. Rhoades for his critical reading of the manuscript and the valuable discussions. Comments by J. Wang and an anonymous reviewer helped to revise this manuscript. The author would like to acknowledge the encouragement he received from K. Aki, and sincerely regrets that $\mathrm{K}$. Aki passed away before this article could be published.

\section{References}

Aki, K., A probabilistic synthesis of precursory phenomena, in Earthquake Prediction, edited by D. W. Simpson and P. G. Richards, pp. 566-574, AGU, 1981.

Daley, D. J. and D. Vere-Jones, An introduction to the Theory of Point Processes, vol. 1, Elementary Theory and Methods, Second edition, 469 pp., Springer, New York, 2003.

Evison, F. F. and D. Rhoades, The precursory earthquake swarm in New Zealand: hypothesis tests II, N. Z. J. Geol. Geophys., 40, 537-547, 1997.

Evison, F. F. and D. Rhoades, The precursory earthquake swarm in Japan: hypothesis test, Earth Planets Space, 51, 1267-1277, 1999.

Geller, R. J., Earthquake predicton: a critical review, Geophys. J. Int., 131, 425-450, 1997.

Grandori, G., E. Guagenti, and F. Perotti, Alarm systems based on a pair of short-term earthquake precursors, Bull. Seismol. Soc. Am., 78, 15381549, 1988 .

Hamada, K., A probability model for earthquake prediction, Earthq. Prediction Res., 2, 227-234, 1983.

Hayakawa, M., O. A. Molchanov, T. Ondoh, and E. Kawai, Anomalies in the sub-ionospheric VLF signals for the 1995 Hyogo-ken Nanbu earthquake, J. Phys. Earth, 44, 413-418, 1996.

Imoto, M., Application of the stress release model to the Nankai earthquake sequence, southwest Japan, Tectonophysics, 338, 287-295, 2001.

Imoto, M., A testable model of earthquake probability based on changes in mean event size, J. Geophys. Res., 108, ESE 7.1-12 No. B2, 2082, doi:10.1029/2002JB001774, 2003.

Imoto, M., Probability gains expected for renewal process models, Earth Planets Space, 56, 563-571, 2004

Imoto, M. and N. Yamamoto, Verification test of the mean event size model for moderate earthquakes in the Kanto region, central Japan, Tectonophysics, 417, 131-140, 2006.

Jones, L., Foreshocks and time-dependent earthquake hazard assessment in southern California, Bull. Seismol. Soc. Am., 75, 1669-1679, 1985.

Katao, H., N. Maeda, Y. Hiramatsu, Y. Iio, and S. Nakao, Detailed mapping of focal mechanisms in/around the 1995 Hyogo-ken Nanbu earthquake rupture zone, J. Phys. Earth, 45, 105-119, 1997.

Mogi, K., Magnitude-frequency relations for elastic shocks accompanying fractures of various materials and some related problems in earthquakes,
Bull. Earthq. Res. Inst., 40, 831-853, 1962.

Mori, J. and R. E. Abercrombie, Depth dependence of earthquake frequency-magnitude distribution in California: Implications for rupture initiation, J. Geophys. Res., 102, 15081-15090, 1997.

Reasenberg, P. and M. Matthews, Precursory seismic quiescence: A preliminary assessment of the hypothesis, Pageoph, 126, 373-406, 1988.

Sakamoto, Y., M. Ishiguro, and G. Kitagawa, Akaike Information Criterion Statistics, D. Reidel, Dordrecht, 290 pp., 1983.

Scholz, C. H., The frequency-magnitude relation of microfracturing in rock and its relation to earthquake, Bull. Seismol. Soc. Am., 58, 399415, 1968.

Schorlemmer, D., S. Wiemer, and M. Wyss, Earthquake statistics at Parkfield: 1. Stationarity of $b$ values, J. Geophys. Res., 109, B12307, doi:10.1029/2004JB003234, 2003.

Schorlemmer, D., S. Wiemer, and M. Wyss, Variations in earthquakesize distribution across different stress regimes, Nature, 437, 539-542, doi:10.1038/nature04094, 2005.

Tsunogai, U. and H. Wakita, Anomalous changes in groundwater chemistry-Possible precursors of the 1995 Hyogo-ken Nanbu earthquake, Japan, J. Phys. Earth, 44, 381-390, 1996.

Utsu, T., Probalities in earthquake prediction, Zisin II, 30, 179-185, 1977 (in Japanese)

Utsu, T., Probabilities in earthquake prediction (the second paper), Bull. Earthq. Res. Inst., 57, 499-524, 1982 (in Japanese).

Vere-Jones, D., Probabilities and information gain for earthquake forecasting, Comput. Seismol., 30, 248-263, 1998.

Wiemer, S. and M. Wyss, Mapping the frequency-magnitude distribution in asperities: an improved technique to calculate recurrence times?, $J$. Geophys. Res., 102, 15,115-15,128, 1997.

Wyss, M., Towards a physical understanding of the earthquake frequencymagnitude distribution, Geophys. J. R. Astron. Soc., 31, 341-359, 1973.

Wyss, M. and D. C. Booth, The IASPEI procedure for the evaluation of earthquake precursors, Geophys. J. Int., 131, 423-424, 1997.

Wyss, M. and S. Matsumura, Mosl likely locations of large earthquakes in the Kanto and Tokai areas, Japan, based on the local recurrence times, Phys. Earth Planet. Interiors, 131, 173-184, 2002.

Yamada, T. and K. Oike, Electromagnetic radiation phenomena before and after the 1995 Hyogo-ken Nanbu earthquake, J. Phys. Earth, 44, 405412, 1996.

Zuñiga, F. R. and M. Wyss, Most- and least-likely locations of large to great earthquakes along the pacific coast of Mexico estimated from local recurrence times based on $b$-values, Bull. Seismol. Soc. Am., 91(6), 1717-1728, 2001

M. Imoto (e-mail: imoto@bosai.go.jp) 